\title{
Internalization of NK cells into tumor cells requires ezrin and leads to programmed cell-in-cell death
}

Shan Wang ${ }^{1,2, *}$, Zhen Guo ${ }^{3, *}$, Peng Xia ${ }^{3}$, Tingting Liu ${ }^{1}$, Jufang $\mathrm{Wang}^{1}$, Shan $\mathrm{Li}^{1}$, Lihua Sun ${ }^{1}$, Jianxin $\mathrm{Lu}^{2}$, Qian Wen ${ }^{4}$, Mingqian Zhou ${ }^{4}, \mathrm{Li} \mathrm{Ma}^{4}$, Xia Ding ${ }^{5}$, Xiaoning Wang ${ }^{1,2,4}$, Xuebiao Yao ${ }^{3}$

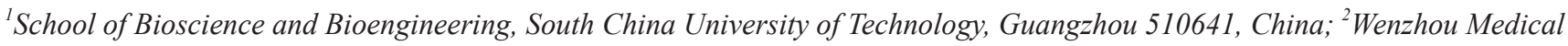
College, Wenzhou 325035, China: ${ }^{3}$ Anhui Key Laboratory for Cellular Dynamics and Chemical Biology, University of Science and Technology of China, Hefei 230027, China: ${ }^{4}$ Nanfang Medical University, Guangzhou 510515, China; ${ }^{5}$ Beijing University of Chinese Medicine, Beijing 100086, China

Cytotoxic lymphocytes are key players in the orchestration of immune response and elimination of defective cells. We have previously reported that natural killer (NK) cells enter target tumor cells, leading to either target cell death or self-destruction within tumor cells. However, it has remained elusive as to the fate of NK cells after internalization and whether the heterotypic cell-in-cell process is different from that of the homotypic cell-in-cell event recently named entosis. Here, we show that NK cells undergo a cell-in-cell process with the ultimate fate of apoptosis within tumor cells and reveal that the internalization process requires the actin cytoskeletal regulator, ezrin. To visualize how NK cells enter into tumor cells, we carried out real-time dual color imaging analyses of NK cell internalization into tumor cells. Surprisingly, most NK cells commit to programmed cell death after their entry into tumor cells, which is distinctively different from entosis observed in the homotypic cell-in-cell process. The apoptotic cell death of the internalized NK cells was evident by activation of caspase 3 and DNA fragmentation. Furthermore, NK cell death after internalization is attenuated by the caspase inhibitor, Z-VAD-FMK, confirming apoptosis as the mode of NK cell death within tumor cells. To determine protein factors essential for the entry of NK cells into tumor cells, we carried out siRNA-based knockdown analysis and discovered a critical role of ezrin in NK cell internalization. Importantly, PKA-mediated phosphorylation of ezrin promotes the NK cell internalization process. Our findings suggest a novel regulatory mechanism by which ezrin governs NK cell internalization into tumor cells.

Keywords: NK cells, tumor cells, entosis, adherent junction, ezrin

Cell Research (2009) 19:1350-1362. doi: 10.1038/cr.2009.114; published online 29 September 2009

\section{Introduction}

The study of cell-in-cell phenomena began with the desire to understand the complex phenotype, which stemmed from Lewis' seminal observation of a nonphagocytotic process of cell-eating-cell in 1925 [1]. Humble et al. [2] coined the term emperipolesis in 1950s, to define the heterogeneous cell-in-cell phenomena when they studied biological interaction of lymphocytes with

\footnotetext{
*These two authors contributed equally to this work. Correspondence: Xiaoning Wang ${ }^{\mathrm{a}}$, Xuebiao Yao

${ }^{a}$ E-mail: xnwang@21cn.net

${ }^{b} E-m a i l: y a o x b @ u s t c . e d u . c n$

Received 19 February 2009; revised 14 June 2009; accepted 16 July 2009; published online 29 September 2009
}

other cells. Since then, emperipolesis has been observed in lymphocyte activities in physiological and pathophysiological settings [3].

In multicellular organisms, the orchestration of cell proliferation and cell death is carefully tuned to control the plasticity of tissues and organs. In the mammary gland, the epithelial cell plasticity and fate are governed by their attachment to extracellular matrix (ECM). Detachment from ECM functions as a means of epithelial cell turnover and glandular luminal clearance during development. Overholtzer et al. [4] have recently described a nonapoptotic cell death mechanism named 'entosis', which is postulated to be involved in human breast cancer development. This entotic cell death mechanism is initiated by an unusual process involving the invasion of one live cell into another, followed by the degradation 
of internalized cells via lysosomal enzymes. Unlike the phagocytic ingestion of apoptotic cells, cell internalization in the entotic process is mediated by adherens junctions. The ROCK activity and actin polymerization of internalizing cells are required for the cell-in-cell process. In addition, internalized cells can be released from target cells or undergo cell division within the target cells, which further distinguishes entosis from phagocytosis. While the cytological features seen in this homotypic cell-in-cell process are well described [4], the underlying molecular mechanisms and the physiological relevance of this homotypic cell-in-cell process have remained elusive.

Interestingly, we had observed heterotypic cell-incell processes similar to that of entotic process about two decades ago when we studied the natural killer (NK) cell biology [5]. In fact, we had carried out classic cytological characterization of the heterotypic cell-in-cell phenomena using NK cell-tumor cell interaction as a model system. Our early studies show that NK cells enter into tumor cells and induce target cell death as well as selfdestruction within tumor cells $[5,6]$. However, our early attempt to elucidate the molecular mechanisms underlying NK cell internalization into tumor cells was hindered by lack of critical tools such as RNA interferencemediated target protein knockdown. Although NK cell internalization into tumor cells exhibits great cytological similarity to that of homotypic cell-in-cell internalization in breast cancer tissue, it remains unclear whether the molecular mechanism underlying homotypic cell-in-cell processes is different from that of heterotypic cell-in-cell processes. In addition, it has remained elusive as to the respective cell fates after NK cell internalization into the target cells.

Ezrin is an actin-binding protein of the ezrin-radixinmoesin family of cytoskeleton-membrane linker proteins involved in a variety of actin-based cellular dynamics [7]. Our previous studies showed that ezrin interacts and codistributes with cytoplasmic $\beta$-actin at the apical membrane of epithelial cells [8]. Recently, we have demonstrated the importance of the phospho-regulation of ezrin in membrane-cytoskeletal remodeling and elucidated how protein phosphorylation regulates the molecular plasticity of ezrin $[9,10]$. Using mouse genetics, Tamura et al. demonstrated the physiological function of ezrin in the animal through knockdown of ezrin to $<5 \%$ of the wild-type levels. Importantly, suppression of ezrin results in severe defects in vesicular membrane trafficking and in plasma membrane-cytoskeletal remodeling of gastric parietal cells, confirming the role of ezrin in orchestrating membrane-cytoskeletal dynamics [11]. Interestingly, ezrin is polarized in uropods of NK-sensitive tumor cells and involved in NK target cell recognition $[12,13]$.

Most studies show that the process of NK killing or entering tumor cells can be separated into three steps, conjugating with target cells, internalization (cell-in-cell process) and initiating killing/executing immuno-modulation after entering the target cells. In pursuit of the molecular mechanism underlying NK cell internalization into tumor cells and to elucidate the molecular pathways underlying heterotypic cell-in-cell event, we tested if NK cell internalization into different types of tumor cells is a cell type-selective process and whether the selectivity is dependent on ezrin expression on target tumor cells. Among various cell lines tested, the A431 cell line exhibited the highest efficiency in attracting NK cells. Since A431 cells contain ezrin-rich microvilli structures [14], and conjugation of NK cells with their cognate target cells requires such membrane protrusive structures, we speculated a potential role of host cell ezrin in NK cell internalization and, therefore, tested the requirement of host cell ezrin in NK cell internalization. Our study revealed a novel role of ezrin in heterotypic cell-in-cell processes.

\section{Results}

NK cells internalize into neighboring target tumor cells following their attachment

To delineate the molecular mechanism underlying NK cell-mediated tumor cell death, we first tested if there is selectivity in NK cell entry into different types of tumor cells. We selected two different types of NK cells, NK92 and NK92L, bearing different cytolytic activities. To further examine the NK cell entry into target tumor cells, NK cells were labeled with CellTracker Green BODIPY and mixed together with tumor cells labeled with CellTracker Red CMTPX in suspension. Our preliminary studies show that the ability of NK cells to enter target cells is independent of their cytolytic potentials (data not shown). Thus, we selected NK92, a less cytolytic cell line, to monitor the internalization process and surveyed the entry of NK92 cell into 14 different lines of tumor cells using real-time imaging (Figure 1A). The internalized cells were confirmed by post-imaging staining (Figure $1 \mathrm{~B}$ and $1 \mathrm{C}$ ). Internalized cells highlighted by fluorescence dyes and DIC images were observed as early as $4 \mathrm{~h}$ after NK92 cells were mixed with target tumor cells in adherent culture and in suspension (data not shown). From two separated preparations using adherent target cells, our analyses of 10 different cell lines show that A431 cells and HCT8 cells are most suitable for studying internalization of NK92 cells due to their morphology and potential in internalizing NK cells (Figure 1D). 
A
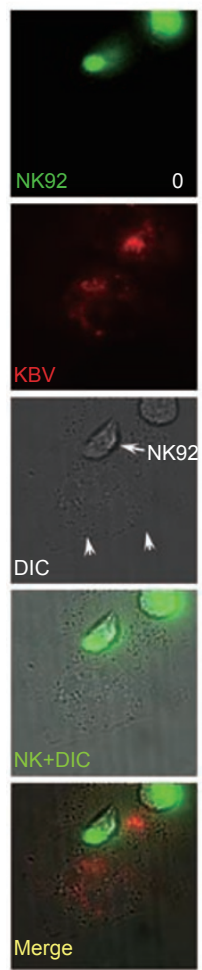
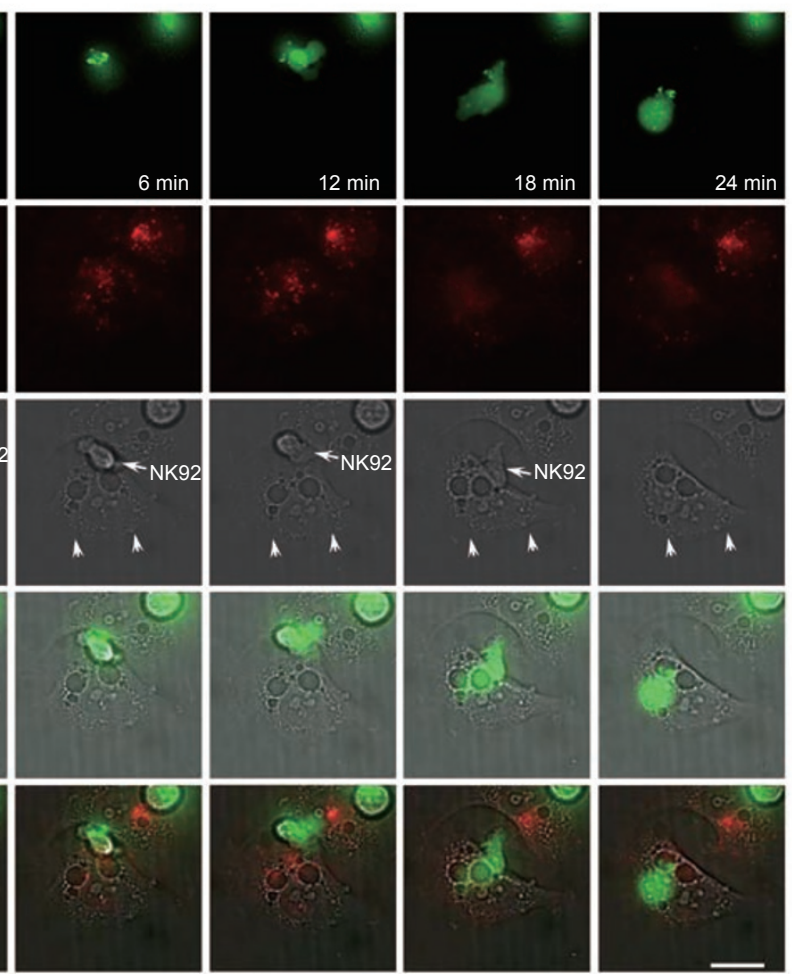
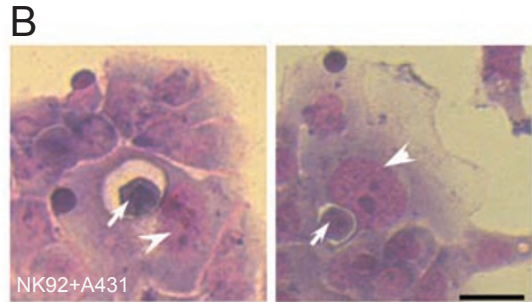

C
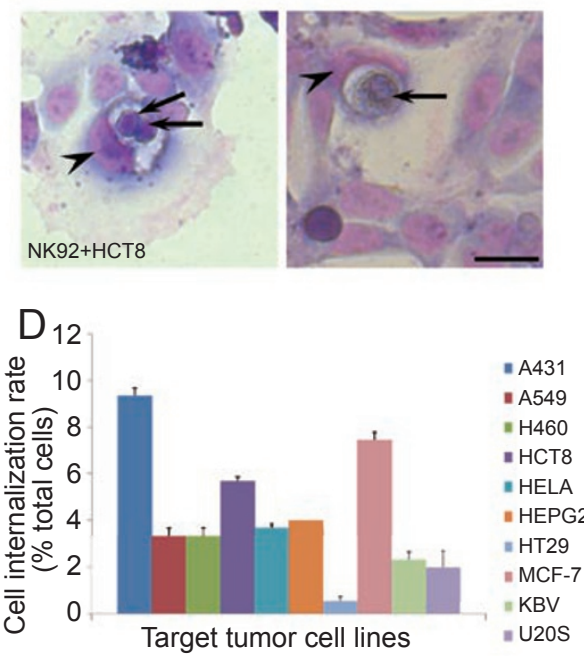

Figure 1 NK cells internalize into neighboring target tumor cells. (A) Real-time imaging of NK92 cell internalization into KBV cells. NK92 cells were labeled with CellTracker Green BODIPY while tumor cells (KBV) were labeled with CellTracker Red CMTPX. After labeling, NK92 cells (pointed by arrows in DIC) were incubated with KBV cells (arrowheads in DIC). Real-time images were collected every $3 \mathrm{~min}$ from three channels. Montages from each individual channel and DIC along with indicated merge are presented. Bar: $10 \mu \mathrm{m}$. (B) NK cell internalization into A431 cells. A431 cells incubated with a NK92 cells for $4 \mathrm{~h}$ followed by fixation and staining with Giemsa. Dark staining marked the nuclei of NK cells (arrows) while tumor cell nuclei were lightly labeled (arrowheads). Bar: $10 \mu \mathrm{m}$. (C) NK cell internalization into HCT8 cells. HCT8 cells incubated with a NK92 cells for $4 \mathrm{~h}$ followed by fixation and staining with Giemsa. Dark staining marked the nuclei of NK cells (arrows) while tumor cell nuclei were lightly labeled (arrowheads). Bar: $10 \mu \mathrm{m}$. (D) Survey of NK cell internalization into a broad spectrum of tumor cells. NK92 cells labeled with CellTracker Green BODIPY were incubated with target tumor cells labeled with CellTracker Red CMTPX. Internalized cells were scored $4 \mathrm{~h}$ after their incubation and expressed as percentage of total cells. About 100 cells from 3 different preparations were counted.

Therefore, we used A431 and HCT8 cells as target cells for further characterization.

NK cell internalization occurred without alteration of targeted tumor cells

To examine if NK cells were completely internalized by target cells, we have carried out confocal microscopic analysis of A431 cells after internalizing NK92 cells. Figure $2 \mathrm{~A}$ projects a three dimensional volume view of an A431 cell with two internalized NK92 cells constructed from 30 optical section series in the z-axis. The three dimensional projection is presented as Supplementary information Movie S1, and the movie confirms that NK92 cells reside within the A431 cell. Thus, we conclude that NK92 cells are internalized into an A431 cell.
To visualize the ultrastructural features of the NK cell within A431 cells, we carried out transmission electron microscopic analyses of serial sections taken from Eponembedded cells. One example of an A431 cell with an internalized NK92 cell was presented in Figure 2B. It is readily apparent that an NK92 cell is contained within a bigger A431 cell that shows a crescent-shaped nucleus (blue arrow), which is pushed to the periphery by the NK92 cell. The A431 cell morphology after internalization of NK92 cells is reminiscent of what was seen in homotypic entosis in breast cancer cells [4]. The transmission electron microscopic analyses demonstrated complete internalization of one cell within another (Figure $2 \mathrm{~B}$, a). It is also apparent that both the NK92 cell nucleus and A431 nucleus are intact, suggesting that NK cell 
A
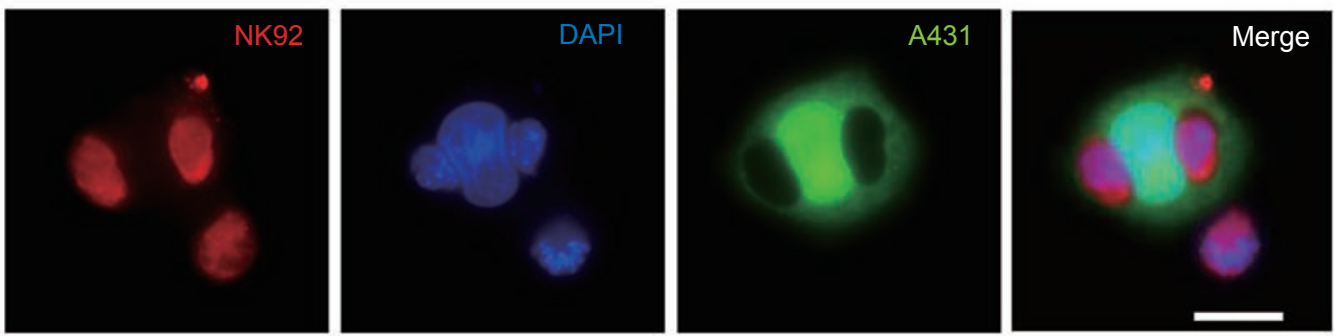

$\mathrm{B}$
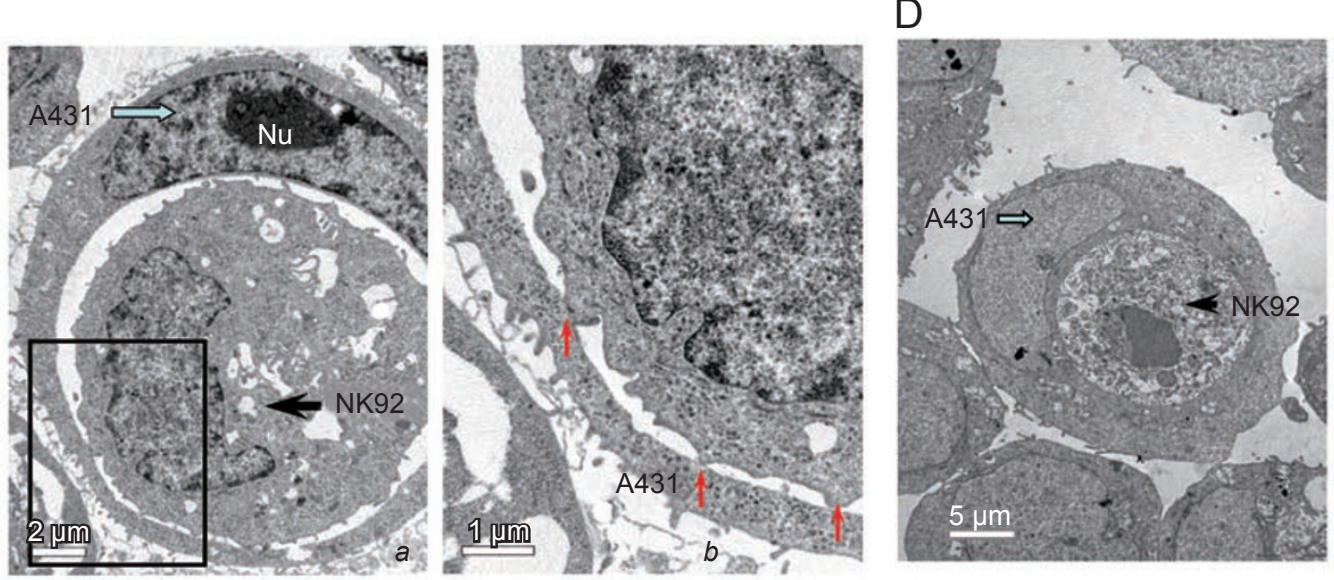

C
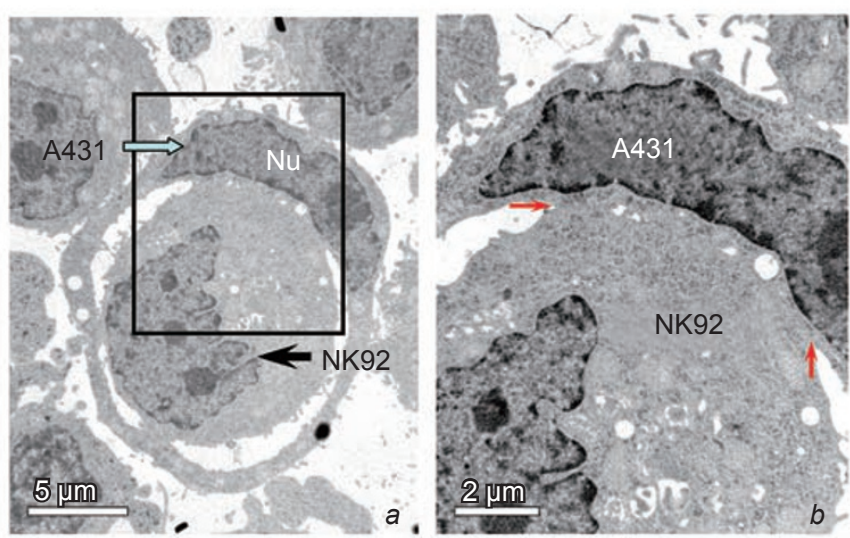

Figure 2 Ultrastructural characterization of NK cell internalization into tumor cells. (A) Three dimensional view of NK92 cell internalization into A431 cells. NK92 cells were labeled with CellTracker Red CMTPX while A431 were labeled with CellTracker Green BODIPY. After labeling, NK92 cells were incubated with A431 cells. Four hours after their incubation, NK cell containing A431 cells were fixed and imaged. Stack images were presented and exported as movie. Bar: $10 \mu \mathrm{m}$. (B) Ultrastructural analyses of A431 cells and internalized NK92 cells. A431 cells were incubated with NK92 cells for $4 \mathrm{~h}$ followed by chemical fixation. Fixed cells were dehydrated and embedded in Epon resin followed by standard electron microscopic specimen processes, as described in Materials and Methods. a, Left panel shows a low power of NK cell-in-A431 cell; NK cell has entered into host cell but they are cytological separated. Bar: $5 \mu \mathrm{m}$. b. Right panel shows magnification of boxed area of Figure 2B. The scale bar is $1 \mu \mathrm{m}$. Smaller arrows indicate membrane contacts between NK cell and A431 cells. Note that both A431 cell nucleus and NK cell nucleus are intact despite the fact that NK92 is completely internalized. (C) Ultrastructural analyses of A431 cells with an internalized NK92 cells. A431 cells were incubated with NK92 cells for $4 \mathrm{~h}$ followed by chemical fixation. a. Fixed cells were dehydrated and embedded in Epon resin followed by standard electron microscopic specimen processes as described in Materials and Methods. b. Magnified area was selected to show the membrane contacts between target cell and a NK cell. Note that A431 cell nucleus is intact while NK cell nucleus appeared tortured. Bar: $2 \mu \mathrm{m}$. (D) Ultrastructural analyses of NK92 cell nuclear disintegration. A431 cells were incubated with NK92 cells for $4 \mathrm{~h}$ followed by chemical fixation. Fixed cells were dehydrated and embedded in Epon resin followed by standard electron microscopic specimen processes, as described in Materials and Methods. While A431 cell nucleus is intact, the NK cell nucleus is fragmented. Bar: $5 \mu \mathrm{m}$. 
entry into target cells occurs independent of cytolysis. Careful examination of the magnified image revealed that NK cell plasma membrane remains connected with A431 cell membrane (Figure 2B, b; red arrows) despite that the NK cell appears residing in a giant vacuole of A431 cell. These contacts of two plasma membranes are reminiscent of adherens junction-like structures seen in breast cancer cell entosis [4]. In some cases, the contacts of two membranes are continuously extended (Figure $2 \mathrm{C}$, red arrows), indicating that two membranes remain interactive after NK cell internalization. Interestingly, after entry into A431 cell, the NK cell nucleus begins to be disintegrated and fragmented (Figure 2D) while A431 cell nucleus remains crescent-shaped and intact, suggesting that NK cell may undergo a nuclear-initiated cell death program after its internalization.

NK cell internalization led to cell death program activation within targeted tumor cells

Recently it was reported that homotypic cell-cell internalization of breast cancer cells leads to a nonapoptotic, lysosome-mediated form of cell death [4]. The irregularity seen in the NK cell nuclei after their internalization prompted us to examine whether the fate of internalization of NK92 cells involves an apoptotic process. The nuclear disintegration and fragmentation prompted us to examine if caspase-mediated cell death accounts for the fate of NK cells after internalization. To this end, we carried out immunocytochemical staining for caspase 3 activity based on the appearance of $17 \mathrm{kDa}$ fragment of cleaved caspase 3, a characteristic marker for caspasedependent cell death [4]. Caspase 3 is mainly localized in the cytoplasm as pro-enzyme but translocated to nucleus upon its activation. As shown in Figure 3 (the merged image), active caspase 3 (red) is located within the hol- low of green fluorescence-labeled (CellTracker) A431 cell. The hollow in green fluorescence channel confirms its absence of A431 constituents. The activation of caspase 3 in the internalized NK cell suggests that NK92 cell death program is activated after its internalization into A431 cell, which is consistent with the ultrastructural analyses of NK cell integrity in the A431 cells. In addition, there were no partially internalized cells that were positive for caspase 3 staining (data not shown), indicating the specificity of apoptotic program commitment by NK cells after their complete entry into A431 cells. We surveyed 120 internalized NK cells from 3 different preparations and found that nearly $90 \%$ of internalized NK cells underwent apoptosis, as judged by activation of caspase 3 (Figure 3B).

If NK cell is engaged in apoptotic processes, irregularity of the NK cell nucleus should be evident due to a typical DNA fragmentation mechanism. To this end, we carried out TUNEL assay to examine the possible DNA fragmentation. As shown in Figure 3C, green fluorescence marked the fragmentation of DNA. Merged images show that the majority of DNA fragmentation occurred within NK cells (red). This apoptotic process was attenuated by the caspase inhibitor Z-VAD-FMK (Figure 3D, $P<0.01)$ but not the negative control of Z-FA-FMK. Thus, we conclude that the NK cells undergo apoptotic cell death after entry into A431 cells.

To validate if the NK cell internalization is an active process, we carried out real-time imaging of NK92 cell entry into A431 cells. To distinguish viable cells from dead ones, NK92 cells were incubated with Annexin VFITC for 15 min prior to its addition into adherent A431 cells. After co-culture for $4 \mathrm{~h}$, time-lapse imaging was collected at intervals of $5 \mathrm{~min}$. As shown in Figure 3E, a viable NK cell (red arrows) underwent dynamic mor-

Figure 3 NK92 cells committed to apoptitic cell death after internalizing into A431 cells. (A) NK cell fate determination after its entry into A431 cells. A431 cells which were labeled with CellTracker Green BODIPY were incubated with NK92 cells for $4 \mathrm{~h}$ followed by fixation and examination of caspase activity using antibody-reacting cleaved caspase 3 (red). Bar: $10 \mu \mathrm{m}$. (B) Survey of NK cell fate after its internalization into A431 cells. NK92 cells marked with red fluorescence were scored while apoptotic cells were counted based on caspase 3 staining. In general, there are $\sim 10 \%$ of A431 cells containing NK92 cells. The internalized cells were expressed as percentage of A431 cells containing NK92 cells while apoptotic NK92 cells were expressed as a percentage of A431 cells containing NK92 cells. About 100 NK92 containing A431 cells were counted from three different preparations. (C) NK cells undergo apoptotic processes after entry into A431 cells. A431 cell internalized NK92 cells were fixed and stained for fragmented DNA (TUNEL, green) to confirm cell fate. Merged image shows that the internalized NK92 cell exhibited typical apoptotic DNA fragmentation. Bar: $10 \mu \mathrm{m}$. (D) Caspase inhibitor Z-VAD-FMK attenuated NK cell death in A431 cells. NK92 cell were pretreated with $5 \mu \mathrm{M}$ Z-VAD-FMK before incubation with A431 cells. Ten hours after their incubation, A431 cells with internalized NK92 cells were fixed and stained for fragmented DNA (TUNEL, green) to confirm cell fate. DMSO was used as a vehicle control and Z-FA-FMK as a negative control. (E) Real-time imaging of NK92 cell internalization into A431 cells. The NK92 cells were labeled with Annexin V-FITC for 15 min before its addition into adherent A431 cell culture for $4 \mathrm{~h}$. Real-time images were collected every 5 min from two channels. Montages from FITC channel and DIC along with indicated merge are presented. Bar: $10 \mu \mathrm{m}$. 
A
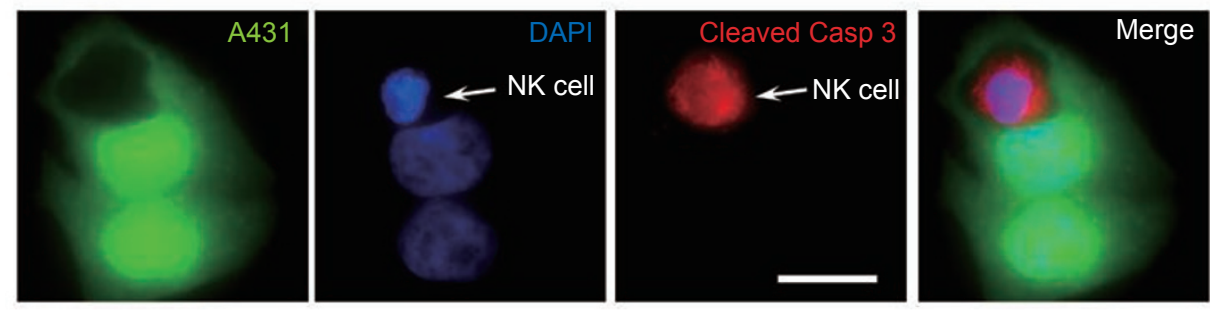

B

D
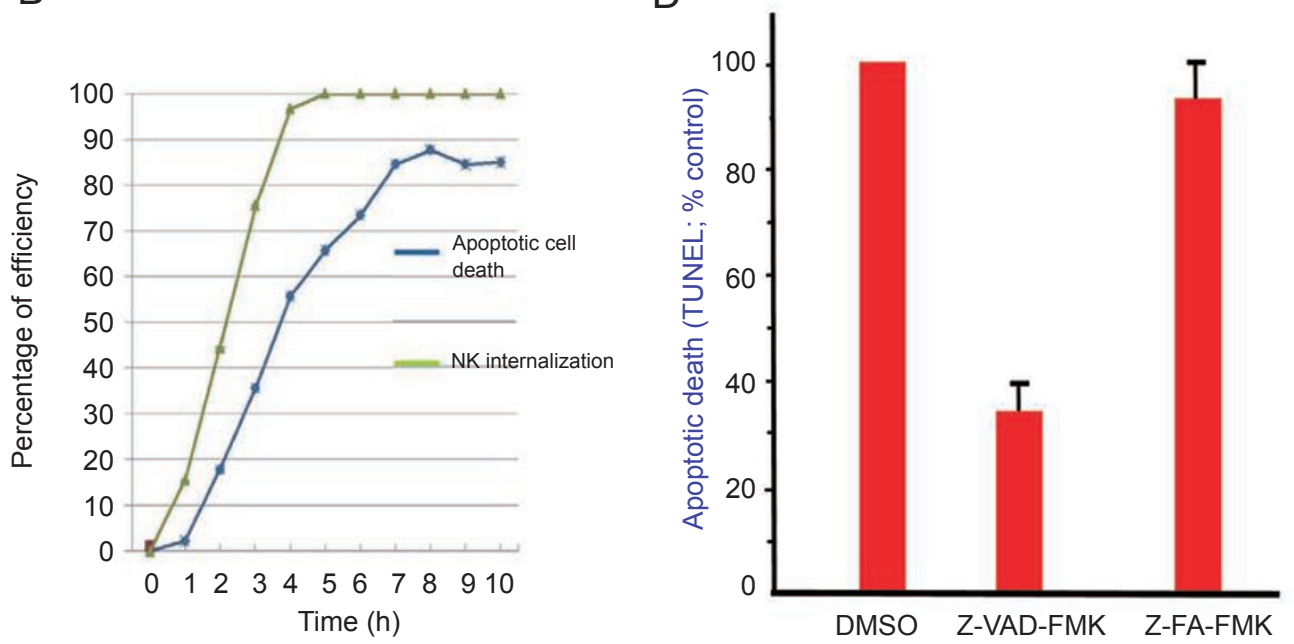

C
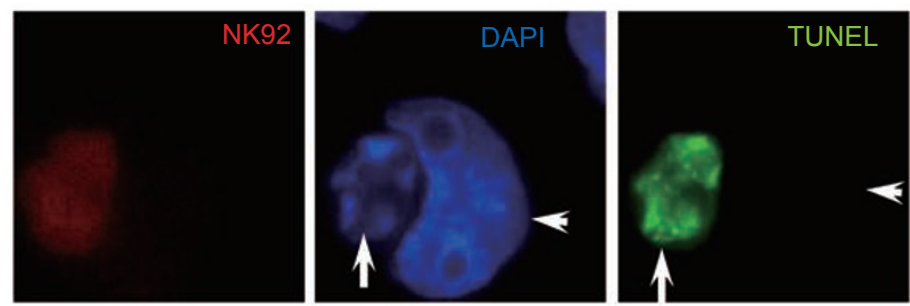

Merge

E
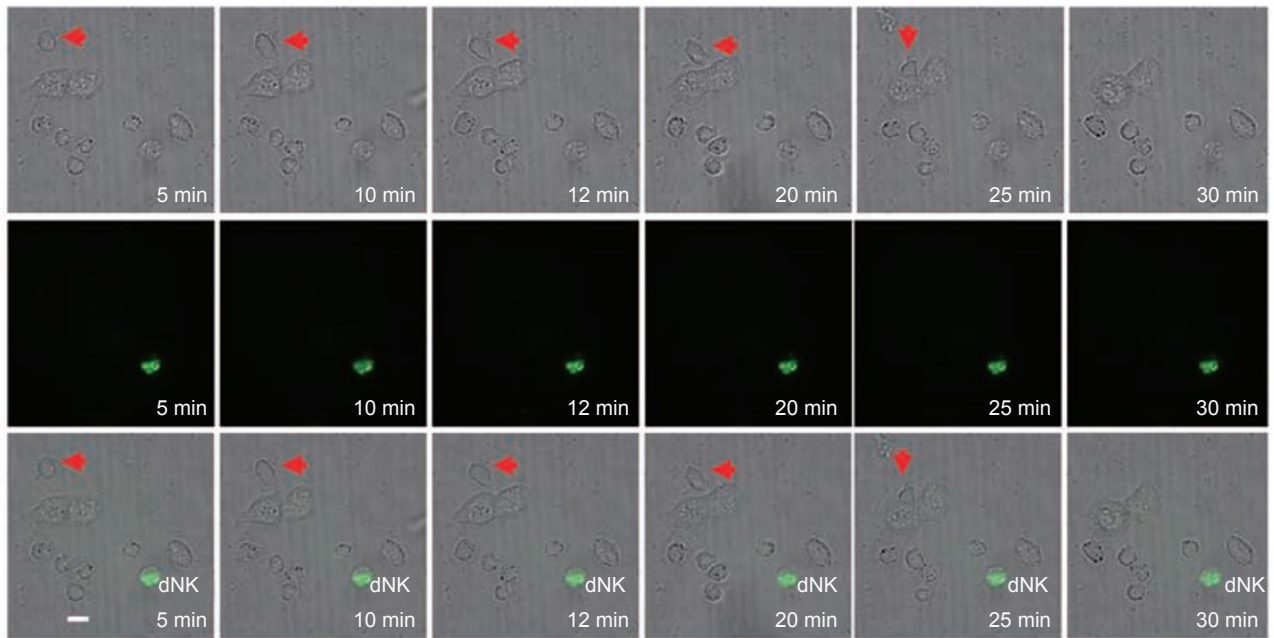
phological changes and then successfully entered into an A431 cell, while an Annexin V-FITC positive dead cell (dNK) remained static during the entire period of imaging (0 30 min), demonstrating that NK cell internalization into A431 cell is an active process. A431 cells do not play any active roles in 'uptaking' dead NK cells. Thus, we conclude that NK cell internalization into tumor cells is an active process requiring NK cell viability.

\section{Internalization of $N K$ cells requires ezrin in A431 cells}

Because both NK92 cells and A431 cells express Ecadherin (Supplementary information, Figure S1), and cells in our time-lapse analysis appeared to establish cellcell junctions prior to NK cell internalization, we examined whether cadherin-mediated adhesion of target tumor cells is required for internalization of NK cells. Cadherin proteins require calcium for binding activity, and thus junctions can be blocked by chelating calcium from the culture medium. Our preliminary studies show that the addition of EGTA (which chelates $\mathrm{Ca}^{2+}$ ) strongly inhibited both conjugate formation and the internalization of NK92 cells into A431 cells (Supplementary information, Figure S2), suggesting that E-cadherin might be essential for NK cell internalization.

To directly examine the requirement of E-cadherin for NK cell internalization into the target cells, we carried out siRNA-mediated knockdown of E-cadherin in A431 cells. The control cells were treated with scramble
siRNA oligonucleotides for $24 \mathrm{~h}$. As shown in Figure 4A, the siRNA specifically knocks down E-cadherin protein levels without alteration of actin and ezrin levels. We then incubated E-cadherin-knockdown A431 cells with NK92 cells, $24 \mathrm{~h}$ after the siRNA treatment, followed by fixation and immunofluorescence staining of F-actin and DNA. As shown in Figure 4B (a), control-transfected cells displayed a typical internalization phenotype as two nuclei are situated in one compartment outlined by F-actin labeling (red) of the plasma membrane (merge). However, knockdown of E-cadherin resulted in an inhibition of cell-cell contact (Figure 4B, b; arrows) and failure in complete internalization of NK92 cell (arrowheads), which is consistent with the notion that E-cadherin is required for bringing two plasma membranes together for zipping $[4,15]$. In fact, knockdown of E-cadherin resulted in inhibition of tumor cell conjugation with NK cells (Figure 4B, b; arrows). Quantitative analyses indicated that reduction of E-cadherin by $87.5 \%$ resulted in an inhibition of $77 \%$ of NK92 cell internalization (Figure $4 \mathrm{C}, P<0.05)$.

Since previous studies demonstrated that exogenous expression of ezrin in NK-resistant cells induces uropod formation and sensitizes target cells to interleukin-2activated killing [12] and ezrin is an important factor in the formation of immunological synapse between the NK cell and target tumor cells [13], we sought to test if repression of ezrin in A431 cells diminishes internaliza-

Figure 4 Ezrin is essential for tumor cells to internalize NK cells. (A) Ezrin siRNA suppresses the ezrin protein accumulation. Aliquots of A431 cells were transfected with ezrin and E-cadherin siRNA oligonucleotides and scramble control as described under Materials and Methods. Cells were then harvested for carrying out SDS-PAGE and subsequent western blotting analyses of the efficiency on suppression of ezrin protein (upper panel) and specificity of this siRNA-mediated ezrin depletion (middle and lower panel). (B) A431 cells were labeled with CellTracker Green BODIPY $36 \mathrm{~h}$ after appropriate siRNA treatment. After labeling, NK92 cells were incubated with A431 cells. Four hours after their incubation, NK cell containing A431 cells were fixed and imaged. Knockdown of E-cadherin prevented NK92 cell-A431 cell contact, while repression of ezrin blocked internalization of NK92 cells. Bar: $10 \mu \mathrm{m}$. (C) Quantification of NK92 cell internalization as function of ezrin. Aliquots of A431 cells were transfected with ezrin and E-cadherin siRNA oligonucleotides and scramble control as described under Materials and Methods. Cells were then incubated with NK92 cells for $4 \mathrm{~h}$ prior to fixation and staining for DNA and F-actin. The internalization was quantified as percentage of A431 cells containing NK92 cells. Internalization data from various treatments are plotted as percentage of the scramble siRNA control for each of experiment. About 100 NK92-positive cells were counted from three different preparations. Error bars represent SE; significant difference from stimulated controls $\left({ }^{*} P<0.05\right)$. (D) Quantification of NK92 cell fate after its internalization into A431 cells. Aliquots of A431 cells were transfected with either ezrin siRNA or E-cadherin siRNA oligonucleotides, as described under Materials and Methods. SiRNA-treated A431 cells were then incubated with NK92 cells for $9 \mathrm{~h}$ prior to fixation and staining for fragmented DNA (TUNEL assay) to confirm cell fate. The cell death rate was quantified as percentage of NK92 cells positive for TUNEL assay in NK92 internalized A431 cells. About 100 NK92 positive cells were counted from three different preparations. Error bars represent SE; significant difference from stimulated controls $(* P<0.05)$. (E) Phosphorylation of ezrin by CAMP-dependent kinase promotes the NK92 cell internalization into A431 cells. Aliquots of A431 cells were treated with $50 \mu \mathrm{M}$ IBMX or $100 \mu \mathrm{M}$ dbcAMP before incubation with NK92 cells. In one case, $10 \mu \mathrm{M} \mathrm{H89}$, a PKA inhibitor, was included in $100 \mu \mathrm{M}$ dbcAMP treatment as described under Materials and Methods. Cells were then incubated with NK92 cells for $4 \mathrm{~h}$ prior to fixation and staining for DNA and F-actin. The internalized cells were quantified as percentage of A431 cells containing NK92 cells. Internalization data from various treatments are plotted as percentage of the DMSO control for each of experiment. About 100 NK92-positive cells were counted from three different preparations. Error bars represent SE; significant difference from stimulated controls $(* P<0.05)$. 
A

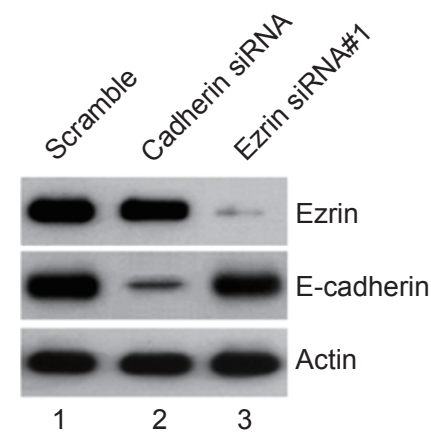

C

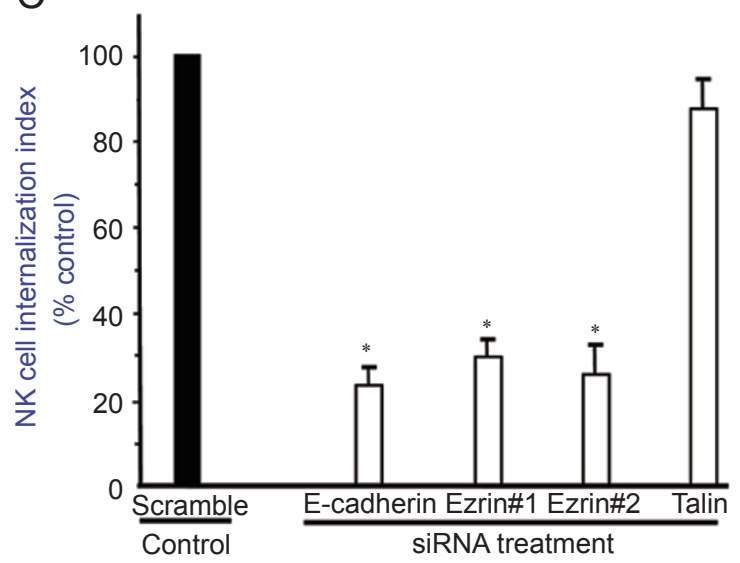

E

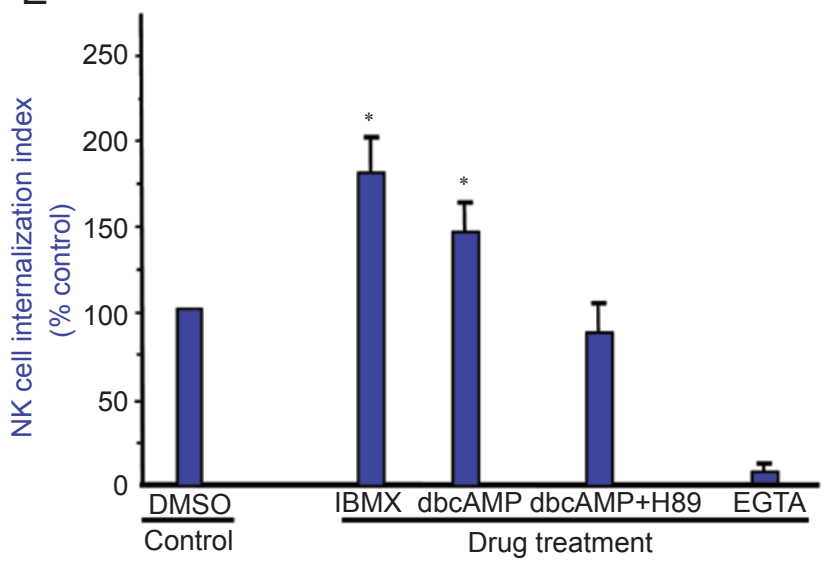

D

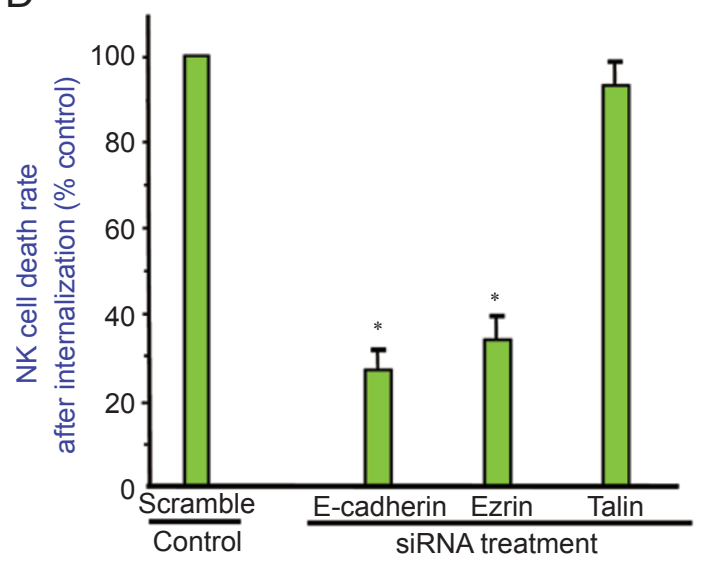


tion of NK cells. To this end, we treated A431 cells with ezrin siRNA oligonucleotides followed by incubation of NK92 cells. Western blotting analyses show that ezrin siRNA caused a specific knockdown of ezrin protein level without altering actin or E-cadherin levels (Figure 4A, lane 3). While suppression of ezrin inhibited a complete internalization of NK cells into A431 cells, the plasma membranes from the NK92 and A431 cells were zipped but not integrated, as evident by the concentration of Factin labeling (Supplementary information, Figure S4, c; arrows). Quantitative analyses indicated that reduction of ezrin by $91.5 \%$ resulted in an inhibition of $71 \%$ of NK92 cell internalization $(P<0.05)$.

To eliminate the potential off-target effect, we carried out another set of knockdown experiment using an siRNA targeting a different region of ezrin. Western blotting analyses indicate that the ezrin siRNA \#2 caused a specific knockdown of ezrin protein level without affecting actin protein level (Supplementary information, Figure S3). To visualize if NK cell internalization depends on ezrin and E-cadherin, we incubated NK92 cells pre-labeled with CellTracker Red with A431 cells that were treated with either E-cadherin siRNA or ezrin siRNA \#2 for $36 \mathrm{~h}$ followed by labeling of CellTracker Green. As shown in Figure 4B, transfection of scramble siRNA does not interfere with the NK cell internalization into A431 cells, as evident by the adjacent position of the two cell nuclei in A431 cell cytoplasm (Figure 4B, a; merge). Careful examination revealed that NK cell nucleus is ruptured after its entry into A431 cells (Figure 4B, a; arrow). Knockdown of E-cadherin prevents NK cell internalization, as E-cadherin is essential for the contact between A431 and NK cells (Figure 4B, b; merge). On other hand, knockdown of ezrin (siRNA \#2) inhibited the complete internalization of NK cells into A431 cells (Figure 4B, c).

The phenotypes seen in the E-cadherin-knockdown cells and ezrin-knockdown cells suggest that ezrin functions in internalization of NK cells into tumor cells while E-cadherin is critical for conjugation of tumor cells with NK cells. To this end, we transfected A431 cells with E-cadherin siRNA oligonucleotide and ezrin siRNA oligonucleotide followed by scoring the efficiency of NK cell death judged by TUNEL assay. As shown in Figure $4 \mathrm{C}-4 \mathrm{D}$, quantitative analyses indicated that reduction of ezrin resulted in inhibition of NK92 cell internalization and reduction in cell death due to insufficiency in NK cell internalization $(P<0.05)$. Suppression of E-cadherin in A431 cells also led to inhibition of NK92 cell internalization and reduction in NK cell death.

Mounting evidence demonstrates that ezrin activity is regulated by site-specific phosphorylation and site-spe- cific phosphorylation governs the spatial localization of ezrin in polarized cells $[11,20]$. If ezrin were important for internalization of NK92 cells, alteration of ezrin plasticity by phosphorylation would modulate NK92-A431 cell interaction. To this end, we treated A431 cells with pharmacological reagents to activate cAMP-dependent protein kinase A followed by incubation with NK92 cells. As shown in Figure 4E, elevation of intracellular cAMP by either dbcAMP or IBMX promotes the efficiency of NK cell internalization. The cAMP-mediated enhancement of NK cell internalization was inhibited by PKA inhibitor H89, suggesting the role of PKA-mediated phosphorylation of ezrin in promoting NK cell internalization. Thus, we conclude that target tumor cell ezrin is essential for internalizing NK cell.

Ezrin-radixin-moesin protein family provides a regulated link between the cortical actin cytoskeleton and the plasma membrane. Phosphorylation of ezrin has been functionally linked to membrane dynamics and plasticity. Our recent study demonstrated that elevation of cAMP leads to activation of PKA and subsequent phosphorylation of ezrin at $\operatorname{Ser}^{66}$ [9]. To verify whether $\operatorname{Ser}^{66}$ of ezrin is phosphorylated in response to dbcAMP treatment, we immuo-isolated ezrin from A431 cells treated with dbcAMP and its control cAMP. Western blotting analysis indicated that ezrin was equally pulled down by the mouse antibody 4A5 (Supplementary information, Figure S5; upper panel). Re-probing of the same blot with an anti-phosph-Ser ${ }^{66}$ rabbit antibody demonstrated that treatment of dbcAMP, but not of cAMP, induced phosphorylation of $\mathrm{Ser}^{66}$, consistent with our early observation that elevation of cAMP in parietal cells induced ezrin phosphorylation and activation [9]. Thus, we suggest that phosphorylation of ezrin at $\mathrm{Ser}^{66}$ facilitates NK cell internalization into A431 cells.

\section{Discussion}

In this report, we extended our early study on NK cell internalization into tumor cells [5], and revealed a critical role of ezrin in NK cell entry into target cell. Surprisingly, while the cytology of NK cell internalization into target cells resembles that of entosis, NK cells undergo an apoptotic death process after being internalized by tumor cells, which is distinctively different from that of entosis. In addition, our study revealed a critical role of tumor cell ezrin in the internalization of NK cell into tumor cells and that PKA-mediated phosphorylation in tumor cells promotes the internalization of NK cells. These findings offer a mechanistic link between the PKAmediated phosphorylation of ezrin and internalization of NK cells by tumor cells. 
Emperipolesis has been found to be commonly enacted by lymphocytes in physiological and pathophysiological settings [16]. Recent reports on the homotypic cell-in-cell process prompted us to examine the internalization process and fate of NK cells after entry into host tumor cells. Our early study revealed the process of NK cell entry into target tumor cells and discovered that internalized NK cells either induced target tumor cell lysis or underwent apoptosis (see Figure 10 in Wang and Li [5]). Due to the limitation of analytic technology, the analyses conducted at that time are limited to cytological and morphological assays. The mechanistic process underlying the fate of NK cells after internalization into tumor cells remained unclear at that time. Survey from our long-term real-time imaging experiment indicates that almost all of the NK cells (>95\%) die at $24 \mathrm{~h}$ after their internalization and the death rate is increased to $98 \%$ at $48 \mathrm{~h}$ after their internalization. Small fraction $(<2 \%)$ of internalized NK cells somehow got released from A431 cells after their initial internalization. While it would be of great interest in the near future to capture those escaped NK cells and compare their molecular signature with that of NK cells that died in A431 cells, our detailed characterization reported here reveals that internalized NK cells ultimately commit to apoptotic cell death via activation of caspase 3 and DNA fragmentation, which is distinctively different from the homotypic cell-in-cell event in entosis.

Natural killer cell internalization by tumor cells may be a mechanism of tumor progression, as cannibalism of the invading cell may provide an advantage to the host tumor cell, perhaps through nutrient recycling during metabolic stress. In fact, Fais and colleagues have found that internalization of $\mathrm{T}$ cells by melanoma cells provides a survival advantage to the tumor cells under conditions of starvation [17]. It has been shown that malignant tumor cells are able to digest internalized cells, both dead and live. However, it has remained elusive whether this internalization of $\mathrm{T}$ cells by melanoma cells represents a typical phenotype seen in malignant tumor cells as a way of surviving in nutrient starvation conditions [18]. Our analyses show that NK cell internalization is a distinct process different from cannibalism because cannibalism is a phagocytotic process in which tumor cells take both viable and dead cells [17]. In contrast, NK cell internalization is an active process and rests on NK cell viability. One important feature of cannibal and malignant cells is their capacity to acidify tumor microenvironment through both lactate accumulation and a progressive $\mathrm{H}^{+}$ concentration increase due to hyperactivity of proton pumps [19]. However, our preliminary experiments show that proton pump inhibitors, such as omperazole, do not modulate NK cell internalization or NK cell survival after internalization (data not shown). In this regard, the NK cell internalization process we observed here is different from that of cannibalism.

As for the functional relevance of NK cell internalization, it is possible that NK cell internalization could promote the development of aneuploidy, as the internalized cells could interfere with tumor cell division by perturbing chromosome segregation and/or cytokinesis. In the future, it will be necessary to examine whether tumor cells with internalized cells exhibit a selective growth advantage and display greater genomic instability using long-term real-time imaging analyses. Given the fact that cytological features seen in the internalization of NK cells into tumor cells exhibit great similarity to those in entosis, it would also be of great interest to test whether the actin cytoskeletal regulator ezrin is essential for entotic processes in tumor cells. Comparative studies of entosis with NK cell internalization into tumor cells will ultimately lead to a better understanding on how cell-incell processes contribute to cell health and diseases.

Phosphorylation of ezrin has been functionally linked to membrane dynamics and plasticity. Our previous study demonstrated that phosphorylation of the conserved $\mathrm{Thr}^{567}$ residue of ezrin alters the physiology of gastric parietal cells [20]. We have recently established a protocol in which phosphorylation-mediated protein conformation can be studied at the single-molecule level [21]. Using this protocol, we have correlated the phosphorylationinduced conformational change of ezrin- $\mathrm{Thr}^{567}$ with its functional activity in cellular localization. Using the same protocol, our preliminary study shows that phoshphorylation of ezrin at $\mathrm{Ser}^{66}$ also unfolds ezrin intramolecular association, suggesting that phosphorylationinduced protein conformational change is used as a signaling mechanism orchestrating cellular dynamics (Wang et al., personal communication). The data presented here reveal that an ezrin-dependent process can mediate NK cell internalization. It has been shown that interleukin-2activated NK cell killing depends upon the intercellular adhesion molecule ICAM-2 and its interaction with ezrin [12]. The level of ICAM-2 expression in NK-sensitive and resistant cells is similar, but in sensitive cells, ICAM-2 is concentrated into bud-like cellular projections known as uropods, whereas in resistant cells, it is evenly distributed. The cytoskeletal-membrane linker protein ezrin is also localized in uropods. Transfection of human ezrin into NK-resistant cells, deficient in ezrin, induces uropod formation, redistribution of ICAM-2 and ezrin, and sensitizes target cells to interleukin-2-activated killing, which is consistent with our finding that ezrin is essential for NK cell internalization. We propose that ezrin 
participates in stabilization of adherent complexes and subsequent remodeling of actin-based cytoskeleton upon cell-cell contact. It is possible that ezrin may provide a spatial cue for the assembly of the protein complex essential for NK cell-A431 cell engagement. It would be of great importance to determine whether internalization of NK cells is a strategy for cancer progression and escape from immuno-surveillance.

Ezrin has been associated with tumor progression in several cancers including the pediatric solid tumors, osteosarcoma and rhabdomyosarcoma [21]. Recent studies suggest that ezrin was dynamically phosphorylated during metastatic progression in osteosarcoma [22]. However, it is currently unclear whether ezrin phosphorylation contributes to the malignancy phenotype of a tumor by promoting tumor cells to internalize NK cells. It would be of great importance to delineate the spatiotemporal dynamics of ezrin phosphorylation related to NK cell internalization. This will require real-time imaging of NK cell internalization into A431 cells expressing a phosphoezrin sensor. In addition, it would be equally important to identify the downstream effector molecules that bind to ezrin in a phosphorylation-dependent manner. Our study shown here aims to provide an outline so that further mechanistic studies of phospho-regulation of ezrin in the NK cell internalization process can be pursued in greater detail.

Taken together, we have characterized NK cell internalization into target cell and revealed that internalized NK cells underwent caspase-dependent cell death. Our finding of phospho-regulation of ezrin by PKA in NK cell internalization demonstrates a critical role of ezrin in cellular dynamics related to the cell-in-cell process. The fact that suppression of ezrin phosphorylation attenuates NK cell internalization into tumor cells and hyper-phosphorylation of ezrin is seen in metastatic tumors points to the importance of ezrin plasticity in cellular health.

\section{Materials and Methods}

\section{Cell culture}

All tested tumor cell lines were purchased from American Type Culture Collection (Manassas, VA, USA). A431, HCT-8 and other 12 cell lines tested were maintained as subconfluent monolayers in DMEM (Invitrogen) with 10\% fetal bovine serum (Hyclone, Logan, UT, USA) and $100 \mathrm{U} / \mathrm{ml}$ penicillin plus $100 \mu \mathrm{g} / \mathrm{ml} \mathrm{strep-}$ tomycin (Invitrogen) at $37^{\circ} \mathrm{C}$ with $10 \% \mathrm{CO}_{2}$. NK92 cells were gifted by Dr Haiming Wei (University of Science \& Technology of China, China).

\section{siRNA treatment and assay for knockdown efficiency}

The siRNA sequences used for silencing ezrin correspond to coding regions 504-524, and the siRNA oligonucleotide was synthesized by Dharmacon. To avoid the off-target effect, an ON-
TARGET plus siRNA for ezrin was obtained from Dharmacon (catalog number: 017370). As a control, either a duplex targeting cyclophilin or a scrambled sequence was used [23]. The siRNA sequence used for silencing E-cadherin was ordered from Dharmacon (catalog number: 003877). In trial experiments, different concentrations of siRNA oligonucleotides were used for different treatment times, as detailed previously, and transfection efficiency was judged based on the uptake of fluorescein isothiocyanateconjugated oligonucleotides [24]. In brief, A431 cells were transfected with ezrin and E-cadherin siRNA oligonucleotides or control scrambled oligonucleotides, and the efficiency of this siRNAmediated protein suppression was judged by western blot analyses.

\section{Immunofluorescence microscopy}

For immunofluorescence, cells after siRNA treatment and subsequent internalization were seeded onto sterile, acid-treated 12$\mathrm{mm}$ coverslips in 24-well plates (Corning Glass Works, Corning, $\mathrm{NY}$, USA). In general, 24-36 h after siRNA transfection, A431 cells were rinsed with Opti-MEM and then incubated with NK92 cells for $4 \mathrm{~h}$ before settling on coverslips. One hour after settling, the cells were fixed in freshly prepared 4\% paraformaldehyde in PBS and rinsed three times in PBS. Cells on the coverslips were blocked with $0.05 \%$ Tween 20 in PBS (TPBS) with $1 \%$ bovine serum albumin (Sigma). These cells were incubated with various primary antibodies in a humidified chamber for $1 \mathrm{~h}$ and then washed three times in TPBS. Filamentous actin was labeled with rhodamine-conjugated phalloidin for outlining general cytology of the plasma membrane while DNA was stained with DAPI (Sigma) for verification of internalization. Coverslips were supported on slides by grease pencil markings and mounted in Vectashield (Vector Laboratories). Images were taken with a Zeiss Axiovert 200 fluorescence microscope using a $63 \times 1.3$ numerical aperture PlanApo objective. Figures were constructed using Adobe Photoshop.

\section{Apoptosis assay}

Annexin V labeling was performed using the Annexin V-FITC Apoptosis Detection Kit I (BD Biosciences \#556547) following the manufacturer's instruction. TUNEL analysis was performed using the DeadEnd Fluorimetric TUNEL System (Promega \#G3250). Cleaved Caspase 3 antibody was purchased from Millipore (\#AB3623) and used as instructed. Percentages of dead cells were calculated out of 200 cells assayed in triplicate. The caspase inhibitor Z-VAD-FMK (catalog number: 550377) and its negative control Z-FA-FMK (catalog number: 550411) were obtained from BD Biosciences (San Jose, CA, USA).

\section{Electron microscopic analyses}

After incubation of NK92 cells with A431 and HCT8 cells for 4 $\mathrm{h}$, the cell mixture was harvested by a brief centrifugation, fixed in $2 \%$ glutaraldehyde and infiltrated according to a standard protocol described previously [5] using LR-gold (Ted Pella Inc, Redding, CA, USA). Thin sections of the LR-gold-embedded glands were picked up on Formvar-coated nickel grids. Grids were stained with $2 \%$ aqueous uranyl acetate for $90 \mathrm{~s}$ and then examined under an electron microscopy.

\section{Time-lapse microscopy}

Time-lapse microscopy was performed as described [25]. Briefly, cells were grown as monolayers on $35-\mathrm{mm}$ coverglass 
bottom dishes (MatTek, Ashland, MA, USA) or in suspension on coverglass bottom dishes coated with polyhema, as described [4]. NK92 cells were labeled with CellTracker Green BODIPY (Invitrogen, SKU\#C2102) while tumor cells were labeled with CellTracker Red CMTPX (Invitrogen, SKU\#C34552) according to the manufacturer's manual. Fluorescence images were obtained every 3 or 6 min for the indicated time courses. For comparison of A431 and NK92 internalized cell fates, all internalized cells were quantified, including those that showed signs of nuclear degradation at the start of the time-lapse. Only internalized cells with nuclei that appeared normal (not degraded or fragmented) at the start of the time-lapse were quantified for cell fate.

\section{Confocal microscopy}

To confirm if NK cells were completely internalized by target cells and avoid out-of-focus artifacts, tumor cells after internalizing NK cells were fixed and examined under a laser-scanning confocal microscope LSM510 NLO (Carl Zeiss) scan head mounted transversely to an inverted microscope (Axiovert 200; Carl Zeiss) with a $40 \times 1.3$ numerical aperture PlanApo objective. Optical section series were collected with a spacing of $0.4 \mu \mathrm{m}$ in the z-axis through $\sim 12-\mu \mathrm{m}$ thickness of the cell-in-cell complex. The images from triple labeling were simultaneously collected using a dichroic filter set with Zeiss image processing software (LSM 5; Carl Zeiss). Digital data were exported into Adobe Photoshop for presentation and as quick-time movie (Supplementary information, Movie S1).

\section{SDS-PAGE and western blotting analyses}

Samples were subjected to SDS-PAGE and transferred onto nitrocellulose membrane. Proteins were probed by appropriate primary antibodies and detected using ECL (Pierce).

\section{Acknowledgments}

We thank members of our group for insightful discussion during the course of this study and Drs Haiming Wei and Zhigang Tian for NK92 cells. This work was supported by grants from National Natural Science Foundation of China (30972681 to XW; 90508002 to XY; 30872286 to LS), Guangdong-NSFC Joint Key Program (to XW), Chinese Academy of Sciences (KSCX1YW-R65, KSCX2-YWH-10), National Basic Research Program of China (973 Program) (2007CB512402 to XW; 2007CB914503 and 2010CB912103 to XY), Ministry of Science \& Technology of China International Collaboration Program (2009DFA31010 to XD), China National Key Projects for Infectious Disease (2008ZX10002-021 to XY), 2007 National Undergraduate Innovative Research Program of China (PX) and KC Wong Education Foundation (ZG).

\section{References}

1 Lewis WH. The engulfment of living blood cells by others of the same type. Anat Rec $1925 ;$ 31:43-49.

2 Humble JG, Jayne WHW, Pulvertaft RJV. Biological interaction between lymphocytes and other cells. Br J Haematol 1956; 2:283-294.

3 Reid FM, Sandilands GP, Gray KG, Anderson JR. Lymphocyte emperipolesis revisited. II. Further characterization of the lymphocyte sub-population involved. Immunol 1979; 36:267372.

4 Overholtzer M, Mailleux AA, Mouneimne G, et al. A nonapoptotic cell death process, entosis, that occurs by cell-incell invasion. Cell 2007; 131:966-979.

5 Wang X, Li W. Mechanisms of natural killer cell-mediated tumor cell cytolysis at a single cell level. J Med Col PLA 1987; 2:107-117.

6 P Xia, S Wang, Z Guo, et al. Emperipolesis, entosis and beyond: dance with fate. Cell Res 2008; 18:705-707.

7 Bretscher A, Edwards K, Fehon RG. ERM proteins and merlin: integrators at the cell cortex. Nat Rev Mol Cell Biol 2002; 3:586-599

8 Yao X, Cheng L, Forte JG. Characterization of the interaction of gastric ezrin with actin. J Biol Chem 1996; 271:7224-7229.

9 Zhou R, Cao X, Watson C, et al. Characterization of PKAmediated phosphorylation of ezrin in gastric parietal cell activation. J Biol Chem 2003; 278:35651-35659.

10 Zhou R, Zhu L, Kodani A, et al. Phosphorylation of ezrin on threonine 567 produces a change in secretory phenotype and repolarizes the gastric parietal cell. J Cell Sci 2005; 118:43814391.

11 Tamura A, Kikuchi S, Hata M, et al. Achlorhydria by ezrin knockdown: defects in the formation/ expansion of apical canaliculi in gastric parietal cells. J Cell Biol 2005; 169:1-8.

12 Helander TS, Carpen O, Turunen O, et al. ICAM-2 redistributed by ezrin as a target for killer cells Nature 1996; 382:265269.

13 Ramoni C, Luciani F, Spadaro F, et al. Differential expression and distribution of ezrin, radixin and moesin in human natural killer cells. Eur J Immunol 2002, 32:11:3059-3065.

14 Hunter T and Cooper JA. Epidermal growth factor induces rapid tyrosine phosphorylation of proteins in A431 human tumor cells. Cell 1981; 24:741-752.

15 Adams CL, Chen YT, Smith SJ, Nelson WJ. Mechanisms of epithelial cell-cell adhesion and cell compaction revealed by high-resolution tracking of E-cadherin-green fluorescent protein. J Cell Biol 1998; 142:1105-1119.

16 Davis DM. Intercellular transfer of cell-surface proteins is common and can affect many stages of an immune response. Nat Rev Immunol 2007; 7:238-243.

17 Lugini L, Matarrese P, Tinari A, et al. Cannibalism of live lymphocytes by human metastatic but not primary melanoma cells. Cancer Res 2006; 66:3629-3638.

18 Fais S. Cannibalism: a way to feed on metastatic tumors. Cancer Lett 2007; 258:155-164.

19 De Milito A, Iessi E, Logozzi M, et al. Proton pump inhibitors induce apoptosis of human b-cell tumors through a caspaseindependent mechanism involving reactive oxygen species. Cancer Res 2007; 67:5408-5417.

20 Liu D, Ge L, Wang F, et al. Single-molecule detection of phosphorylation-induced plasticity changes during ezrin activation. FEBS Lett 2007; 581:3563-71.

21 Khanna C, Wan X, Bose S, et al. The membrane-cytoskeleton linker ezrin is necessary for osteosarcoma metastasis. Nat Med 2004; 10:182-186.

22 Ren L, Hong SH, Cassavaugh J, et al. The actin-cytoskeleton linker protein ezrin is regulated during osteosarcoma metastasis by PKC. Oncogene 2009; 28:792-802. 
23 Cao X, Ding X, Guo Z, et al. PALS1 specifies the apical localization of ezrin in gastric prietal cells. J Biol Chem 2006; 280:13584-13592.

24 Yao X, Zheng Y, Sullivan KF, et al. CENP-E forms a link between attachment of spindle microtubules to kinetochore and the mitotic checkpoint. Nat Cell Biol 2000; 2:484-491.

25 Yuan $\mathrm{K}, \mathrm{Hu} \mathrm{H}$, Guo Z, et al. Phospho-regulation of HsCDC14A by polo-like kinase 1 is essential for mitotic progression. J Biol Chem 2007; 282:27414-27423.

(Supplementary information is linked to the online version of the paper on the Cell Research website.) 